\title{
Influenza Epidemic After the 2011 Great East Japan Earthquake and Tsunami
}

\author{
Jun Namiki, MD, Shun Kohsaka, MD, and Rihito Ui, MD
}

W e report our experience from the Great East Japan Earthquake and Tsunami of March 11, 2011, at a temporary clinic in a large regional gymnasium. From March 19, a total of 19877 survivors who lost their homes after the tsunami were sheltered in evacuation facilities in the Kesennuma city of Sanriku-a region of northeastern Japan. The evacuation shelter, called the $\mathrm{K}$-wave gymnasium, housed approximately 1800 survivors. ${ }^{1}$ The K-wave had a unique design; it was composed of 3 buildings that were connected by corridors - the main building, Kyudo-jo, and Budo-jo (Figure 1). The main building had a large arena of approximately $2000 \mathrm{~m}^{2}$, smaller subarena that was used as a mortuary, and some meeting rooms that we used one of those as a clinic.
In the days and weeks after the devastating natural disasters, the threat of infectious disease outbreak was high. ${ }^{2}$ In addition to the cold weather, risks occurred from poor hygiene due to the disrupted water supply and inadequate air ventilation (Figure 2). On our first day at the facility (March 21), we identified a febrile girl as the first person with influenza in the Kesennuma region, using the rapid antigen test. A number of control measures were promptly implemented. The patient and her immediate family members were moved to a locker room in the Budo-jo and treated with anti-influenza drugs.

On March 22, a febrile boy was diagnosed with influenza $\mathrm{A}$; he, too, was treated and moved into the

\section{FIGURE 1}

\section{A Satellite Image of the K-Wave Gymnasium.}

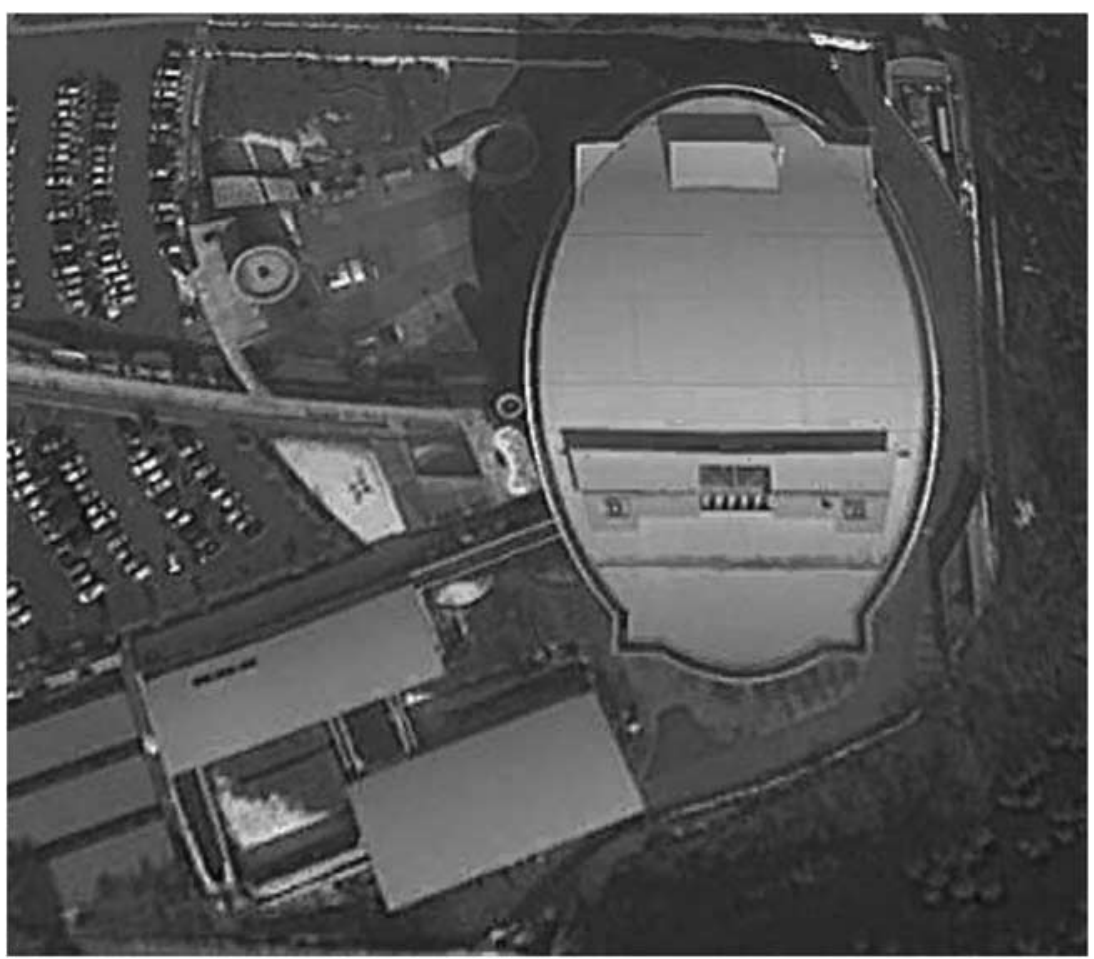

The main (oval) building is connected to the Kyudo-jo (rectangular building, middle left) by a corridor, and the Budo-jo (rectangular building, bottom left) is next to and connected to the Kyudo-jo by another corridor. From Google Earth/( 2012 ZENRIN, Image $\odot 2012$ GeoEye. 
Evacuees Were Housed in the Main Arena of the Main Building.

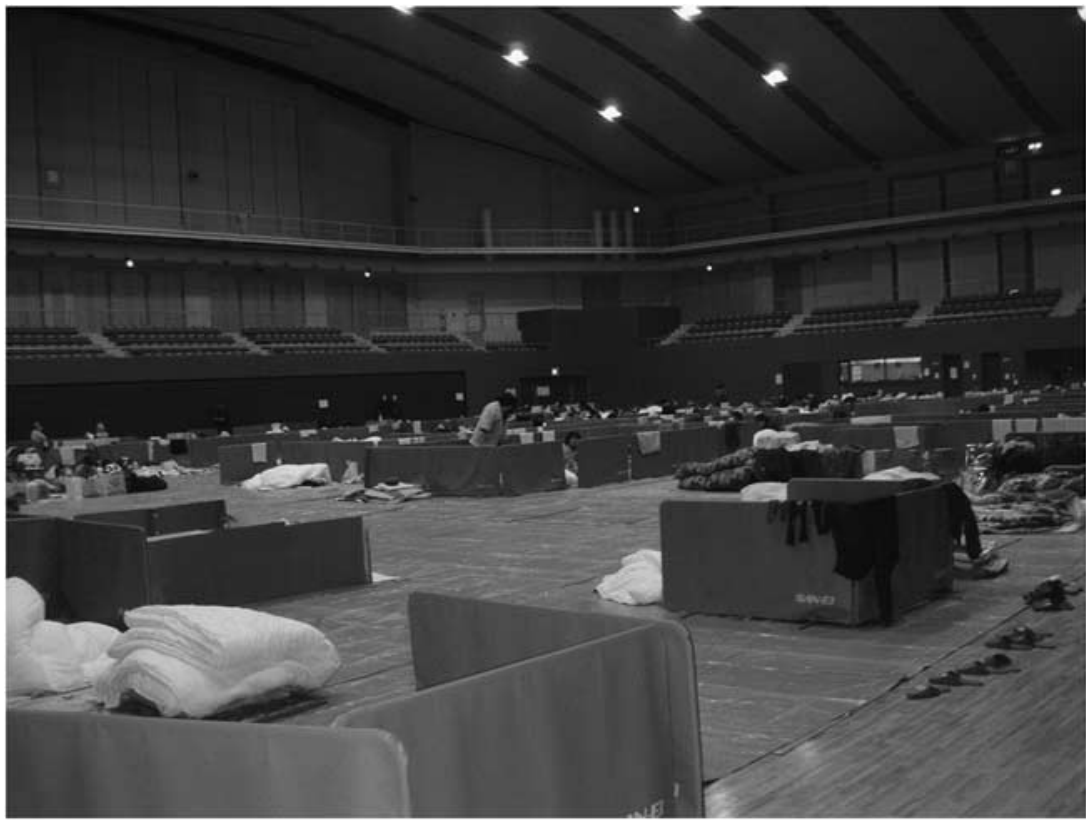

isolation room in the Kyudo-jo. Subsequently, we started to triage all febrile patients. By the next day (March 23), which was our last day in charge of the facility, the situation had worsened. We were informed by a physician who worked overnight that another 4 patients were positive for influenza A and had been treated and isolated in the Kyudo-jo.

At this point, we received permission from the superintendent of the $\mathrm{K}$-wave to move the evacuees from the Kyudo-jo to the other buildings. These evacuees were thus separated from the patients in the Kyudo-jo building. We then set up a second Kyudo-jo clinic for febrile patients to keep the patients with complaints other than fever separated from febrile patients at the main building clinic. One patient at the Kyudo-jo clinic was positive for influenza A.

During the 3 days, a total of 7 febrile patients tested positive for influenza A with use of the rapid antigen test. Thereafter, the influenza epidemic in the K-wave resolved, although the clinic diagnosed 8 additional patients through March 27. If it were necessary to isolate patients with distinct infectious diseases or a large number with influenza, the Budo-jo building could have been used as another isolation facility.

A large regional gymnasium should be available for potential use as an evacuation shelter in case of disasters. In coastal regions, such facilities must be built far enough inland to avoid the impact from tsunamis. The $\mathrm{K}$-wave was located $66 \mathrm{~m}$ above sea level and approximately $3 \mathrm{~km}$ inland from the Kesennuma Bay-a distance that evacuees from coastal communities might reach. The design of the $\mathrm{K}$-wave gymnasium also demonstrated how the 2 satellite buildings that were connected to building by corridors helped to isolate patients with infectious diseases. Also, it would be ideal to have such buildings equipped with pressurized/depressurized air conditioning to isolate survivors and patients from an epidemic of infectious diseases. However, the presence of corridors (about $25 \mathrm{~m}$ between buildings) could maintain an isolated atmospheric environment in each building, even if ventilation were inadequate due to an electrical power outage. The corridors also could provide comfortable transfer paths between the buildings for patients, other evacuees, and the medical and relief personnel. We believe that the design of the $\mathrm{K}$-wave gymnasium contributed to curtailing an influenza epidemic after the tsunami.

\section{About the Authors}

Departments of Emergency and Critical Care Medicine (Dr Namiki), Internal Medicine (Dr Kohsaka), and Ophthalmology (Dr Rihito), Keio University School of Medicine, Tokyo, Japan.

Address correspondence and reprint requests to Jun Namiki, MD, 35 Shinanomachi, Shinjuku-ku, Tokyo 160-8582, Japan (e-mail: namikijun@a8.keio.jp).

\section{Acknowledgments}

Staffs of the supporting team, Keio University Hospital, for logistics. Etsuko Abe, RN, and Kazunari Isogami, B Pharm, Keio University Hospital, for nursing and medication. Medical personnel from various districts in Japan for volunteerism.

\section{Supplementary materials}

To view supplementary material for this article, please visit $h t t p: / / d x$.doi.org/ $10.1017 / d m p .2013 .15$

Published online: April 3, 2013. 


\section{REFERENCES}

1. Kohsaka S, Endo Y, Ueda I, et al. Necessity for primary care immediately after the March 11 tsunami and earthquake in Japan. Arch Intern Med. 2012;172:290-291.
2. Connolly MA, Gayer M, Ryan MJ, et al. Communicable diseases in complex emergencies: impact and challenges. Lancet. 2004;364:1974-1983.

3. Hatta M, Endo S, Tokuda K, et al. Post-tsunami outbreaks of influenza in evacuation centers in Miyagi Prefecture, Japan. Clin Infect Dis. 2012;54:e5-e7. 